\title{
Assessing circadian rhythms in propofol PK and PD during prolonged infusion in ICU patients
}

\author{
Agnieszka Bienert • Krzysztof Kusza • \\ Katarzyna Wawrzyniak • Edmund Grześkowiak • \\ Zenon J. Kokot • Jan Matysiak • Tomasz Grabowski • \\ Anna Wolc $\cdot$ Paweł Wiczling $\cdot$ Miłosz Regulski
}

Received: 12 January 2010/Accepted: 29 May 2010/Published online: 11 June 2010

(C) The Author(s) 2010. This article is published with open access at Springerlink.com

\begin{abstract}
This study evaluates possible circadian rhythms during prolonged propofol infusion in patients in the intensive care unit. Eleven patients were sedated with a constant propofol infusion. The blood samples for the propofol assay were collected every hour during the second day, the third day, and after the termination of the propofol infusion. Values of electroencephalographic bispectral index (BIS),
\end{abstract}

\author{
A. Bienert $(\bowtie) \cdot$ E. Grześkowiak · M. Regulski \\ Department of Clinical Pharmacy and Biopharmacy, Karol Marcinkowski University of Medical \\ Sciences, ul. Marii Magdaleny 14, 61-861 Poznań, Poland \\ e-mail: agnbienert@op.pl \\ K. Kusza · K. Wawrzyniak \\ Department of Anaesthesiology and Intensive Therapy, Collegium Medicum in Bydgoszcz, \\ Nicolaus Copernicus University in Torun, ul. Marii Skłodowskiej-Curie 9, 85-094 Bydgoszcz, \\ Poland \\ K. Kusza \\ Chair of Anaesthesiology and Intensive Therapy, Karol Marcinkowski University of Medical \\ Sciences, ul. Marii Magdaleny 14, 61-861 Poznań, Poland
}

\section{Z. J. Kokot · J. Matysiak}

Department of Inorganic and Analytical Cemistry, Karol Marcinkowski University of Medical Sciences, ul. Grunwaldzka 6, 60-780 Poznań, Poland

T. Grabowski

Center of Pharmacokinetics Research "FILAB”, RAVIMED Sp. z o.o., ul. Polna 54, 05-119 Łajski, Legionowo
A. Wolc
Department of Genetics and Animal Breeding, Poznań University of Life Sciences, ul. Wołyńska 33, 60-637 Poznań, Poland
P. Wiczling
Department of Biopharmaceutics and Pharmacodynamics, Medical Univeristy of Gdańsk, ul. Hallera 107, 80-401 Gdańsk, Poland 
arterial blood pressure, heart rate, blood oxygen saturation and body temperature were recorded every hour at the blood collection time points. A two-compartment model was used to describe propofol pharmacokinetics. Typical values of the central and peripheral volume of distribution and inter-compartmental clearance were $V_{C}=27.71, V_{T}=8011$, and $C L_{D}=2.731 / \mathrm{min}$. The systolic blood pressure (SBP) was found to influence the propofol metabolic clearance according to $\mathrm{Cl}$ $(1 / \min )=2.65 \cdot(1-0.00714 \cdot(\mathrm{SBP}-135))$. There was no significant circadian rhythm detected with respect to propofol pharmacokinetics. The BIS score was assessed as a direct effect model with $E C_{50}$ equal $1.98 \mathrm{mg} / \mathrm{l}$. There was no significant circadian rhythm detected within the BIS scores. We concluded that the lightdark cycle did not influence propofol pharmacokinetics and pharmacodynamics in intensive care units patients. The lack of night-day differences was also noted for systolic blood pressure, diastolic blood pressure and blood oxygenation. Circadian rhythms were detected for heart rate and body temperature, however they were severely disturbed from the pattern of healthy patients.

Keywords Propofol - Circadian rhythm · Pharmacokinetics · Pharmacodynamics · Sedation monitoring

\section{Introduction}

Both undersedation and oversedation have an obvious effect on morbidity and mortality in critically ill patients [1]. The electroencephalographic (EEG) bispectral index (BIS) has been shown to be a quantifiable measure of the hypnotic effect of anesthetic drugs on the central nervous system [2-4]. A correlation between BIS values and the administrated dose of intravenous and inhalational anesthetics has been demonstrated: the progressive deepening of anesthesia induces a corresponding progressive reduction in BIS values [5]. Doi et al. showed a good correlation between BIS, propofol blood concentration and clinical sedation criteria in patients undergoing minor surgery [6]. Similar results were obtained in the study of Lee in respect to propofol effect site concentration and BIS values [7]. Park et al. [8] observed moderate correlation (Spearman's coefficient $r=-0.559$ ) between BIS values and predicted plasma concentration of propofol during recovery from propofol anesthesia in children.

In the literature different data exist with respect to the utility of BIS for monitoring prolonged sedation especially in the critically ill or mechanically ventilated patients. Data on BIS monitoring in the intensive care unit (ICU) are controversial. Leslie et al. [9] noted that BIS decreases linearly as propofol blood concentrations increase in healthy male volunteers. Simmons et al. [10] studied 62 mechanically ventilated patients and demonstrated good correlation between BIS and the SAS (Sedation-Agitation Scale), however the correlation varied in medical, surgical and trauma patients. Riker et al. [11] found good correlation between BIS and the SAS although the authors noted that electromyographic interference could affect the accuracy of BIS. Frenzel et al. [12] examined the correlation between BIS values and different clinical depth of sedation scales (OAAS, modified Ramsay 
scale, modified Glasgow Coma Scale, Cook Scale, and Sedation-Agitation Scale) in 19 patients who required mechanical ventilation for more than $24 \mathrm{~h}$. The authors noted moderate correlation between BIS and each sedation score in 11 patients and no correlation in 8 patients. They found no parameters distinguishing between these two groups. Riess et al. [13] noted that although BIS correlates with Ramsay sedation score in critically ill patients, electromyographic activity and the patients' temperature instability increases BIS values.

In critically ill patients, the alterations of pharmacokinetics and a high interpatient variability of the pharmacodynamic effect can be seen. A common complication of sedation is tolerance, which can modify the concentration-effectrelationship [14]. When a drug in prolonged infusion is administered, circadian rhythms also have to be taken into account as factors influencing both pharmacokinetics and pharmacodynamics. Animal studies have shown that the maximum hypnotic effect of general anesthetics is generally obtained during the rest phase [15]. Challet et al. [16] recently demonstrated that the duration of propofol anesthesia in rats exhibits a three-fold amplitude variation, depending on the hour of administration. The longest duration occurred during the natural sleep period (30 vs. $10 \mathrm{~min}$ during the activity phase). The authors did not measure blood concentrations in this study. It is possible that their observations resulted partly from circadian fluctuations in pharmacokinetic properties. However, such fluctuations in propofol pharmacodynamics should also be taken into consideration. It has been observed that sedative effect of propofol is mediated by $\mathrm{GABA}_{\mathrm{A}}$ receptors in an endogenous sleep pathway [17]. No literature data reflecting propofol chronopharmacokinetics and chronopharmacodynamics in humans exist.

The aim of our study was to propose pharmacokinetic (PK) and pharmacodynamic (PD) models of propofol and to examine the circadian rhythms in propofol concentrations, sedative effects as well as the time-dependent changes in the monitored physiological parameters during prolonged propofol infusion in ICU patients.

\section{Materials and methods}

\section{Patients}

The study was performed with approval of the local Ethics Committee in 11 ICU patients of both genders. The exclusion criteria were: administration of neuromuscular blockers, head trauma and known neurological disorders that might alter EEG interpretation: metabolic or hypoxic encephalopathy, meningoencephalitis, stroke, tumors and perinatal neurologic disorders. Bispectral Index was measured using an EEG monitor (BIS, Monitor Intelli Vue MP60, Philips). Patients had conventional monitoring with at least electrocardiographic monitoring, arterial blood pressure monitoring, body temperature monitoring, and pulseoximetry. Patients were studied in the absence of stimulation when they were clinically stable. Vasopressors had not been given during the study period. 
All the patients were sedated with propofol (Perfusion Space Pump, Braun, Germany) for more than $24 \mathrm{~h}$, and the infusion rate was constant during the study period, except patient 6, who required changes in the infusion rate. Patient 3 was excluded from analysis since no blood samples were collected. The initial propofol dose was $1.5 \mathrm{mg}$ per $\mathrm{kg}$ followed by maintenance doses of $0.7-0.9$ $\mathrm{mg} / \mathrm{kg} / \mathrm{h}$. Blood samples for the propofol assay were collected from the patients' artery every hour during the second and third day of infusion and then 3, 5, 10, $15,30,60,120,180,240 \mathrm{~min}$ after termination of the propofol infusion. The values of BIS, systolic (SBP) and distolic (DBP) blood pressure, heart rate (HR), blood oxygen saturation (OXYG) and body temperature (TEMP) were recorded every hour at the blood collection time points. The blood samples were transferred into heparinised tubes and they were centrifuged immediately after collection. The plasma was stored at $4^{\circ} \mathrm{C}$. The propofol concentration in plasma was measured within eight weeks by high-performance liquid chromatography with a fluorescence detector [18]. The limit of quantification was estimated at $0.01 \mathrm{mg} / \mathrm{l}$. The within-day coefficients of variation and the between-day coefficients of variation were less than $10 \%$.

\section{Population pharmacokinetic modeling}

Population nonlinear mixed-effect modeling was done using NONMEM (Version 6.1.0, Icon Development Solutions, Ellicott City, MD, USA) and the Intel Fortran Compiler 9.0. NONMEM runs were executed using Wings for NONMEM (WFN611, http://wfn.sourceforge.net). The first-order conditional estimation with interaction (FOCE) method was used. The minimum value of the NONMEM objective function $(M O F)$, typical goodness-of-fit diagnostic plots, and an evaluation of the precision of pharmacokinetic parameter and variability estimates were used to discriminate between various models during the model-building process.

\section{PK/PD model of propofol}

The propofol concentration in the plasma versus time (t) was described by a twocompartment model (ADVAN3, TRANS4):

$$
\begin{aligned}
& V_{C} \frac{d C_{P}}{d t}=\text { Input }-C l \cdot C_{P}-C l_{D} \cdot C_{P}+C l_{D} \cdot C_{T} \\
& V_{T} \frac{d C_{T}}{d t}=C l_{D} \cdot C_{p}-C l_{D} \cdot C_{T}
\end{aligned}
$$

where $C_{P}$ and $C_{T}$ are the propofol concentrations in the central and peripheral compartment, $V_{C}$ and $V_{T}$ denote the volumes of the central and peripheral compartment, and $C l$ and $C l_{D}$ denotes the metabolic and inter-compartmental clearance. The input reflects the infusion rate and all extra boluses that were given to individual patients.

The time course of electroencephalographic (EEG) bispectral index (BIS) has been described by a direct effect model: 


$$
B I S(t)=B I S_{0}-\frac{E_{\max } C_{P}}{E C_{50}+C_{P}}
$$

where $B I S_{0}$ denotes the baseline BIS score (fully awake). It was fixed to 100 . The $E_{\text {max }}$ is the maximal effect also fixed to 100 and $E C_{50}$ is the concentration for $50 \%$ decrease in BIS score.

Inter-individual variability (IIV) for the PK/PD parameters was modeled assuming log normal distribution:

$$
P=\theta_{P} \exp \left(\eta_{P}\right)
$$

where $P$ is the individual parameter, $\theta_{P}$ is the typical value of this parameter in the population, and $\eta_{P}$ is a random effect for that parameter with the mean 0 and variance $\omega_{P}^{2}$. The observed concentration of propofol and BIS score were defined by the following equations:

$$
\begin{gathered}
C_{P, o b s}=C_{P}\left(1+\varepsilon_{p r o p, C}\right) \\
B I S_{o b s}=B I S\left(1+\varepsilon_{p r o p, B I S}\right)
\end{gathered}
$$

where $C_{P}$ and BIS are defined by the Eq. 1 and 2 of the basic structural population model and $\varepsilon_{\text {prop,C }}$ and $\varepsilon_{\text {prop,BIS }}$ represents the proportional residual random errors of propofol concentrations and BIS scores. It was assumed that $\varepsilon$ is normally distributed with the mean of 0 and variances denoted by $\sigma_{p r o p, C}^{2}$ and $\sigma_{p r o p, B I S}^{2}$.

Covariate search and rhythm assessment

Covariate search was performed by plotting individual estimates of the PK/PD parameters against time independent covariates (body weight, age, and initial triglyceride concentration) to identify their influence. Similarly the time-dependent covariates were identified by plotting the weighted residuals of the simple model against the covariate. The time-dependent covariates were heart rate, systolic and diastolic blood pressure, body temperature and blood oxygenation. The following regression model was used:

$$
P=\left(\theta_{P 1}+\theta_{P 2}(C O V-\overline{C O V})\right) \exp \left(\eta_{P}\right)
$$

where the $\theta_{P 1}$ and $\theta_{P 2}$ are the regression coefficients. The variables were centered around their median values, $\overline{C O V}$, thus allowing $\theta_{P 1}$ to represent the parameter estimate for the typical patient.

The major objective of this work was to seek the presence of the circadian changes in propofol concentrations, BIS scores, and physiological parameters routinely monitored during patient stays in an ICU. For that purpose the metabolic clearance, $E C_{50}$, heart rate, systolic and diastolic blood pressure and body temperature were sought to be time-dependent according to the cosine function:

$$
P=\theta_{P}\left(1+\theta_{A, P} \cos \left(\frac{2 \pi}{24}\left(t-\theta_{S, P}+T\right)\right)\right) \exp \left(\eta_{P}\right)
$$


where $P$ denotes the studied parameter $\left(C l, E C_{50}\right.$, HR, TEMP, OXYG, SBP, DBP), $\theta_{P}$ is a mesor (the average value around which the variable oscillates), $\theta_{A, P}$ is amplitude, $\theta_{S, P}$ is the acrophase (the clock time at which the peak of a rhythm occurs), and $T$ is the clock time at the start of infusion. The equation assumes a $24 \mathrm{~h}$ fixed period and coverts time to radians.

The difference in the minimum of the NONMEM objective function (MOF) obtained for the two hierarchical models (likelihood ratio) is approximately $\chi^{2}$ distributed. During the covariate and rhythm search the effect of each covariate was examined by adding Eq. 6 or 7 to the base model. The likelihood ratio was determined as the difference in the objective function (MOF) of a full model (with covariate or with daily fluctuations) and reduced model (without covariate or without daily fluctuations) after refitting the data. The difference in $M O F$ between models of 10.8 for one degree of freedom and of 13.8 for two degrees of freedom was considered to be statistically significant at $P<0.001$ for the equation to be included into the base model.

\section{Results}

The patient characteristics are presented in Table 1. Eight male and three female patients were enrolled. The mean duration of the total propofol infusion was $98.4 \pm 84.5 \mathrm{~h}$. The mean duration of the propofol infusion during sample collection (second and third day of infusion) was $38.17 \pm 10.98 \mathrm{~h}$ and the mean steady-state propofol concentration was $0.48 \pm 0.21 \mathrm{mg} / \mathrm{l}$.

The model building process started with a 3-compartment model according to literature findings $[19,20]$. However, a two-compartment model was sufficient to describe our data. The propofol PK during and after prolonged infusion is presented in Fig. 1. This plot shows that the final PK model describes the measured concentrations well. The parameter estimates obtained by the fitting of the PK data are listed in Table 2. The typical value of the volume of the central compartment

Table 1 Demographic data and physiological parameters of subjects

\begin{tabular}{ll}
\hline Parameter, unit & Mean \pm SD $(n=11) ;$ range \\
\hline Age, years & $63 \pm 11 ; 53-85$ \\
Body weight, kg & $72.61 \pm 22.79 ; 50-95$ \\
Height, cm & $168.33 \pm 8.66 ; 150-175$ \\
Body mass index & $25.45 \pm 7.02 ; 17.3-33.2$ \\
Male/female & $8 / 3$ \\
Duration of the infusion & $38.17 \pm 10.98 ; 25-49$ \\
$\quad$ study period, h & $135.0 \pm 19.4 ; 85-191$ \\
Systolic blood pressure, $\mathrm{mmHg}$ & $64.6 \pm 10.0 ; 38-98$ \\
Diastolic blood pressure, $\mathrm{mmHg}$ & $89.8 \pm 15.7 ; 60-138$ \\
Heart rate, beats/min. & $37.0 \pm 0.5 ; 35.7-38.2$ \\
Body temperature, ${ }^{\circ} \mathrm{C}$ & $97.4 \pm 2.1 ; 90-100$ \\
Blood oxygen saturation, $\%$ & \\
\hline
\end{tabular}


ID 1

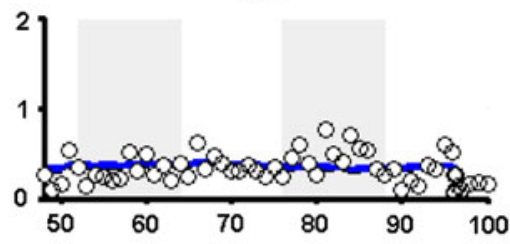

ID 4

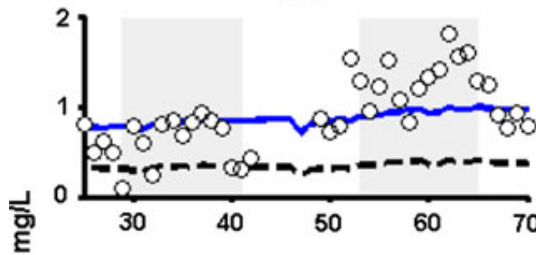

ID 6

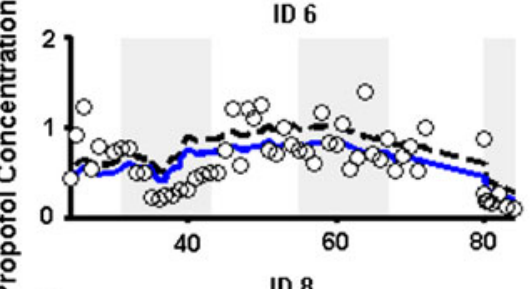

ID 8

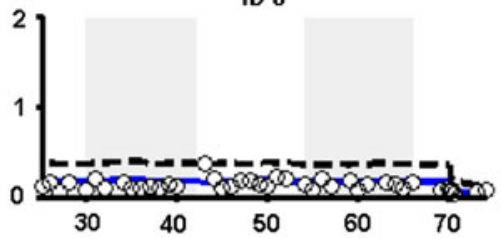

ID 10

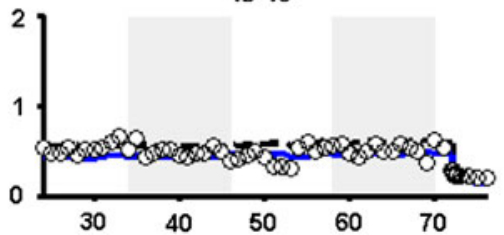

ID 2

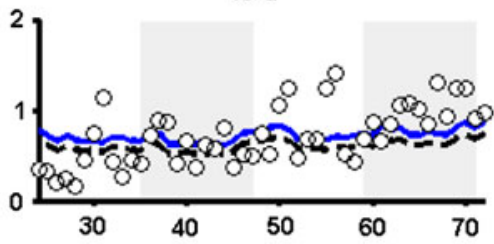

ID 5

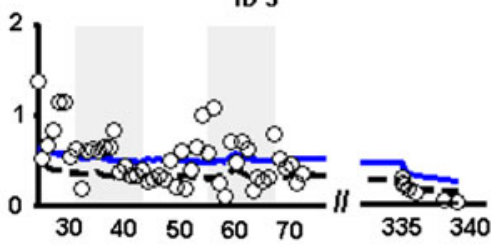

ID 7

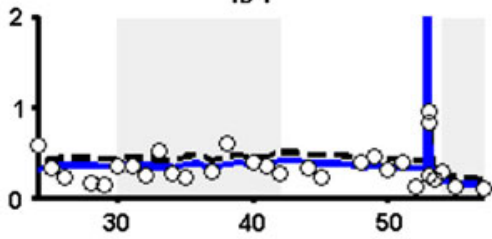

ID 9

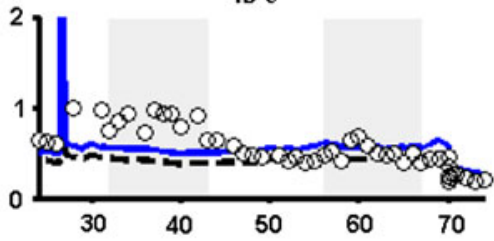

ID 11

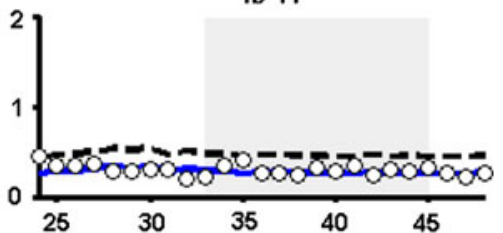

Time, $\mathbf{h}$

Fig. 1 Plot of observed (open circle), population predicted (dashed line) and individual predicted (Solid line) propofol concentrations versus time for the final PK model. The shaded area represents the dark period ( 8 p.m. -8 a.m). Time denotes time that has passed since the start of infusion

was 27.71 . The volume of the peripheral compartment was much higher 8011 . The metabolic clearance for typical subject equaled $2.65 \mathrm{l} / \mathrm{min}$. It was found to depend on the systolic blood pressure according to $C L(1 / \mathrm{min}))=2.65 \cdot(1-0.00714$. (SBP - 135)). This was the only covariate found to influence the model performance with a decrease of 20.3 in MOF. The inter-compartmental clearance equaled $2.73 \mathrm{l} / \mathrm{min}$. The selection of the IIV parameters was done according to the bottom-up approach starting from clearance, as it drives the steady-state concentration, and 
Table 2 Summary of the final population PK parameters, inter-subject and residual error variance estimates of propofol

\begin{tabular}{lll}
\hline Parameter, unit & $\begin{array}{l}\text { Basic PK model } \\
\text { Estimate }(\% \mathrm{CV})\end{array}$ & $\begin{array}{l}\text { PK model with covariate } \\
\text { Estimate }(\% \mathrm{CV})\end{array}$ \\
\hline Fixed effect & & \\
$\theta_{C l},(1 / \mathrm{min})$ & $2.55(16)$ & $2.65(15) \cdot(1-0.00714(66) \cdot(\mathrm{SBP}-135)$ \\
$\theta_{V C}(1)$ & $27.2(24)$ & $27.7(27)$ \\
$\theta_{C L D}(1 / \mathrm{min})$ & $2.70(24)$ & $2.73(28)$ \\
$\theta_{V T}(1)$ & $785(35)$ & $801(33)$ \\
Inter-individual variability & & $48.6(50)$ \\
$\omega_{C l}^{2}(\%)$ & $52.2(50)$ & $41.8(17)$ \\
Residual variability & & \\
$\sigma_{\text {prop }, C p}^{2}(\%)$ & $42.5(21)$ & $-1105.4(\Delta \mathrm{MOF}=-20.3)$ \\
Performance measures & & \\
$M O F$ & -1085.1 &
\end{tabular}

SBP denotes systolic blood pressure

expanding the set parameters until failure in the estimation process was observed. The IIV of clearance equaled $48.6 \%$. We failed to estimate IIV for any other parameters. The relative standard errors $(\% \mathrm{CV})$ of all estimates did not exceed $66 \%$.

The pharmacodynamic model was based on the sigmoid $E_{\max }$ model presented in the literature $[21,22]$. The BIS score data along with model predictions are presented in Fig. 2. These plots show that the PD model described the data reasonably well. There are some parts of the data that are mis-predicted. This might be a consequence of some irregular awakening and sedation periods that were not included into the model, i.e., the daily care of patients by nurses or a short nap. The parameter estimates of the PD model are listed in Table 3 . The $E C_{50}$ equaled $1.98 \mathrm{mg} / \mathrm{l}$ with IIV of $45.1 \%$. The parameters were estimated with high precision. The relative standard error was lower than $36 \%$. No covariates were identified for propofol PD.

Neither for the PK or PD of propofol did we observe circadian rhythms. The inclusion of the daily fluctuations in clearance or $E C_{50}$ did not improve the basic model performance. The MOF decreased by 4.9 for $C L$ and by 13.5 for $E C_{50}$, which was not statistically significant $(P<0.001)$.

Significant circadian rhythms were found for the heart rate $(P<0.001)$ and body temperature $(P<0.001)$. The mesor, amplitude and acrophase estimates are given in Table 4. The time course of body temperature and heart rate along with model predictions (Eq. 7) is presented in Figs. 3 and 4. The heart rate and body temperature were adequately described by the proposed model, however some mispredictions can be observed for temperature. The average heart rate was 90.5 beats/ min with moderate IIV of $14.7 \%$. The amplitude was small and equaled $4.8 \%$ of the messor. The acrophase was $14 \mathrm{~h}$ reflecting an increased heart rate during the day with maximum at 02:00 p.m. The average body temperature was $37^{\circ} \mathrm{C}$ with low IIV of $0.82 \%$. The amplitude was very small, $0.32 \%$ of the mesor. The acrophase was $21.4 \mathrm{~h}$ (21:24 p.m.). The lack of statistically significant circadian rhythms was observed with respect to SBP, DBP and blood oxygen saturation. 
ID 1

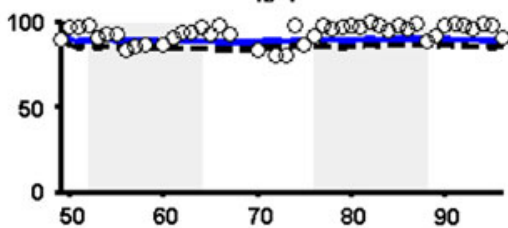

ID 4

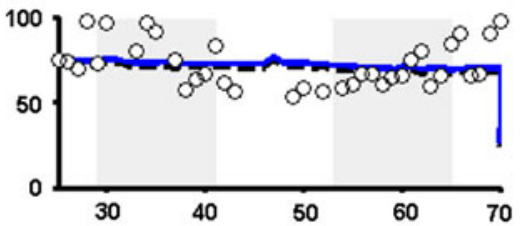

ID 6

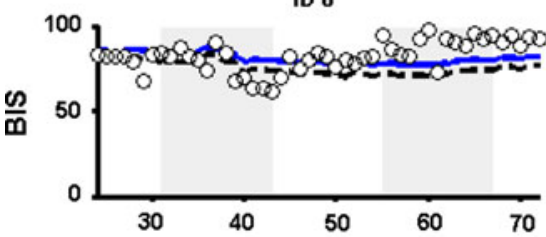

ID 8

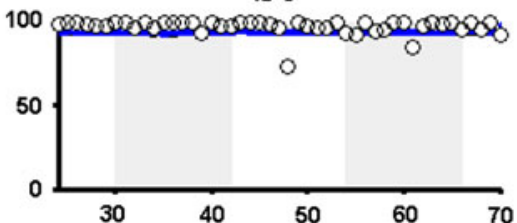

ID 10

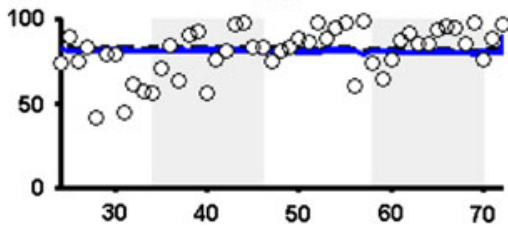

ID 2

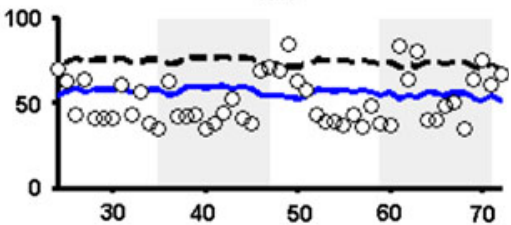

ID 5

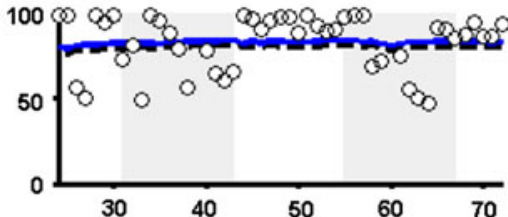

ID 7

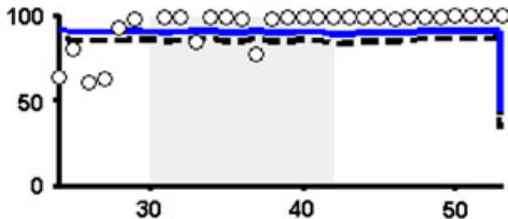

ID 9

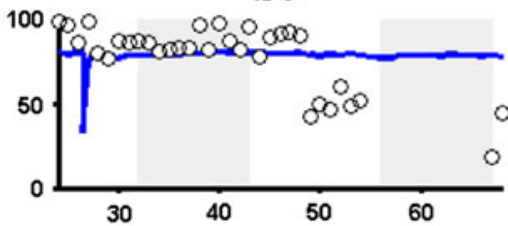

ID 11

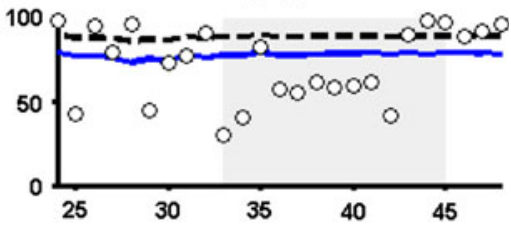

Time, $\mathrm{h}$

Fig. 2 Plot of observed (open circle), population predicted (dashed line) and individual predicted (solid line) BIS scores versus time for the final PD model. The shaded area represents the dark period (8 p.m.-8 a.m). Time denotes time that has passed since the start of infusion

\section{Discussion}

We assessed circadian rhythms during propofol constant infusion in critically ill patients. A three-compartment model has been widely used in studying propofol PK $[19,20]$. However, in this work the presence of the third compartment was not justified, since it manifests itself at the first minutes after the end of infusion or bolus administration whereas our post-infusion data collection started at $3 \mathrm{~min}$. The applied two-compartment model was also used for propofol PK, especially for 
Table 3 Summary of the final population PD parameters, intersubject and residual error variance estimates of BIS scores
Parameter, unit

Basic PK model Estimate (\% CV)

\begin{tabular}{ll}
\hline Fixed effect & \\
$\theta_{E C 50}, \mathrm{mg} / \mathrm{l}$ & $1.98(20 \%)$ \\
$\theta_{E M A X}$ & 100 (Fixed) \\
$\begin{array}{l}\text { Inter-individual variability } \\
\omega_{E C 50}^{2}[\%]\end{array}$ & $45.1(37 \%)$ \\
$\begin{array}{l}\text { Residual variability } \\
\sigma_{\text {prop }, C p}^{2}[\%]\end{array}$ & $19.1(28 \%)$ \\
Performance measures & \\
MOF & 2647.1
\end{tabular}

Table 4 The biological rhythms in physiological parameters with statistically significant circadian rhythms monitored in 11 ICU patients sedated with prolonged infusion of propofol

\begin{tabular}{llll}
\hline $\begin{array}{l}\text { Physiological } \\
\text { parameter } \\
\text { (Symbol) }\end{array}$ & Parameter, unit & Basic model & Basic model with rhythm \\
\hline Heart rate (HR) & $\theta_{H R}($ beats/min) & $90.5(4.7)$ & $90.5(4.7)\left(1+0.048(36) \cos \left(\frac{2 \pi}{24}(t-14(3.6)+T)\right)\right)$ \\
& $\omega_{H R}^{2}(\%)$ & $14.4(34)$ & $14.7(34)$ \\
& $\sigma_{\text {prop }, H R}^{2}(\%)$ & $12.4(44)$ & $11.9(42)$ \\
& $M O F$ & 2513.893 & $2482.476(\Delta \mathrm{MOF}=-31.417)$ \\
Body temp. & $\theta_{\text {TEMP }}\left({ }^{\circ} \mathrm{C}\right)$ & $37.0(0.3)$ & $37(0.3)\left(1+0.00326(52) \cos \left(\frac{2 \pi}{24}(t-21.4(9.5)+T)\right)\right)$ \\
$($ TEMP) & $\omega_{\text {TEMP }}^{2}(\%)$ & $0.816(38.6)$ & $0.823(38)$ \\
& $\sigma_{\text {prop }, T E M P}^{2}(\%)$ & $0.0996(22)$ & $0.966(19)$ \\
& $M O F$ & -395.163 & $-418.75(\Delta \mathrm{MOF}=-23.587)$ \\
\hline
\end{tabular}

critically ill patients [21-24]. The estimated value of metabolic clearance $2.65 \mathrm{l} / \mathrm{min}$ was in agreement with literature data obtained in ICU patients, viz. $2.11 \mathrm{l} / \mathrm{min}$ [25], $1.57 \mathrm{l} / \mathrm{min}$ [26], $1.50 \mathrm{l} / \mathrm{min}$ [27], $1 \mathrm{l} / \mathrm{min}$ [19], $2.2 \mathrm{l} / \mathrm{min}$ [21] and $2.05 \mathrm{l} / \mathrm{min}$ [22]. Similarly the volume of distribution at steady-state $\left(V_{S S}=V_{P}+V_{T}=8281\right)$ was in agreement with literature data, namely 16991 [25], 4991 [28], 11601 [22], 1901 [21], 45771 [27], however $\mathrm{V}_{\mathrm{SS}}$ varies considerably across studies. Our results are also comparable to the results of a multicenter population study [19]. A threecompartment model gave the following parameters for a $70-\mathrm{kg}$ adult: metabolic clearance $1.44 \mathrm{l} / \mathrm{min}$, inter-compartmental clearances 2.25 and $0.92 \mathrm{l} / \mathrm{min}$, volume of the central compartment 9.31 and peripheral compartments 44.2 and 2661 .

In this work we identified the systolic blood pressure as a covariate affecting metabolic clearance. Propofol is highly extracted drug due to its hepatic metabolism. This results in dependence of clearance on liver blood flow. It has been shown that the rate of propofol clearance increased with the higher liver blood flow, resulting in lower propofol concentrations in blood [24]. In the present study we observed that the increased SBP led to decreased clearance. However, it is difficult to draw any conclusion about the possible mechanism, since there is no 
ID 1

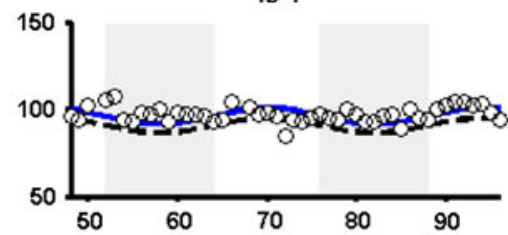

ID 4

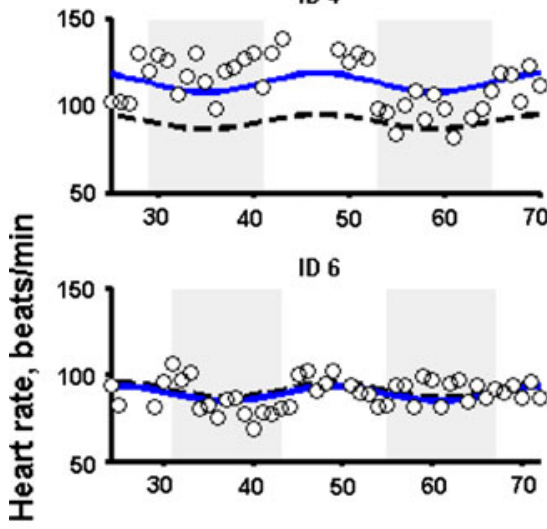

ID 8

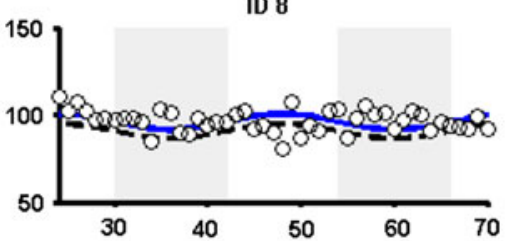

ID 10

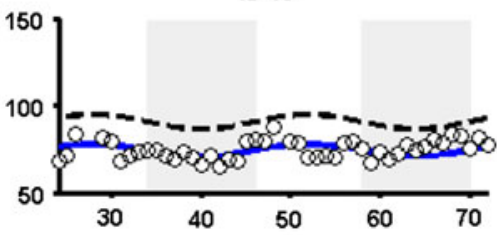

ID 2

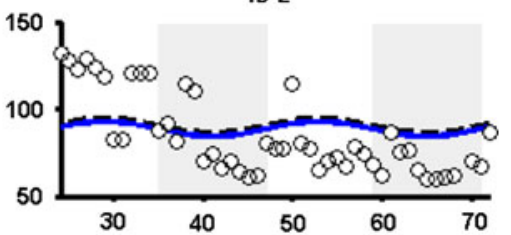

ID 5

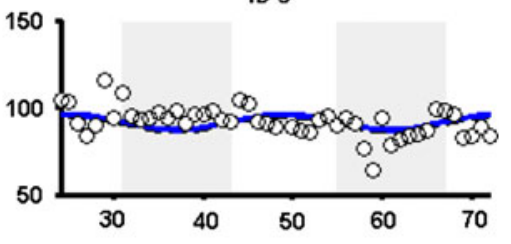

ID 7

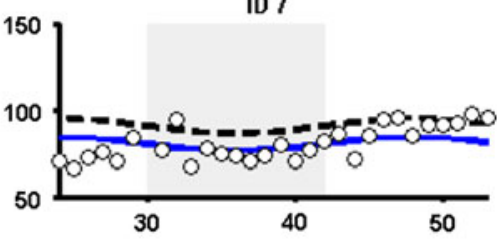

ID 9

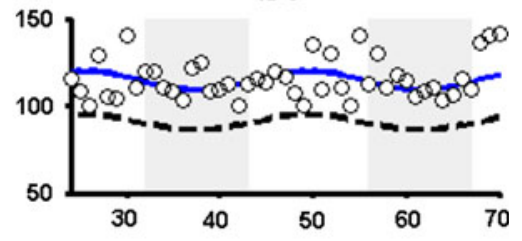

ID 11

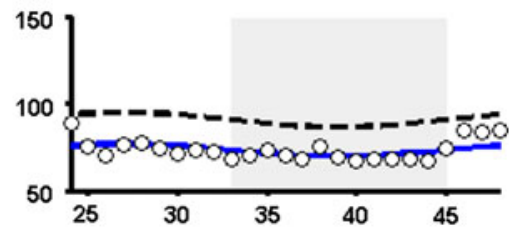

Time, $\mathrm{h}$

Fig. 3 Plot of observed (open circle), population predicted (dashed line) and individual predicted (solid line) hear rates versus time for the final model (Eq. 7) with circadian rhythms. The shaded area represents the dark period ( 8 p.m. -8 a.m). Time denotes time that has passed since the start of infusion

direct relationship between liver blood flow and SBP, especially in critically ill patients. In the literature, in contradiction to our findings, both temperature and triglyceride concentration was found to influence the metabolic clearance [21].

The utility of BIS monitoring during prolonged sedation in critically ill patients is still discussed and the literature data is mostly based on the correlation between different sedation scores $[10,12,13]$. However, it is routinely used to monitor degree of anesthesia or sedation. Our results suggest the there is a direct link 
ID 1

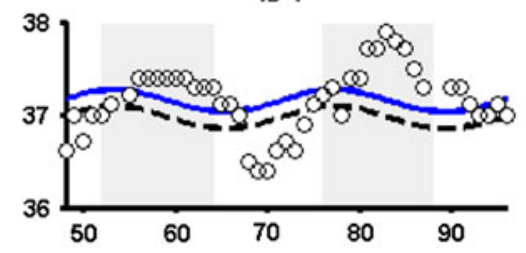

ID 4

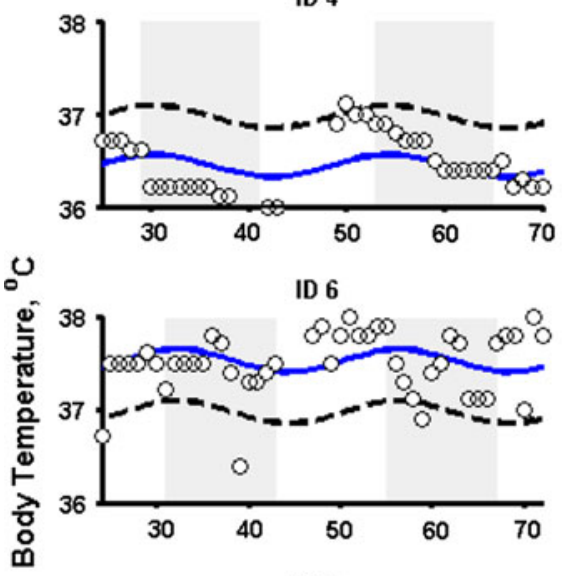

ID 8

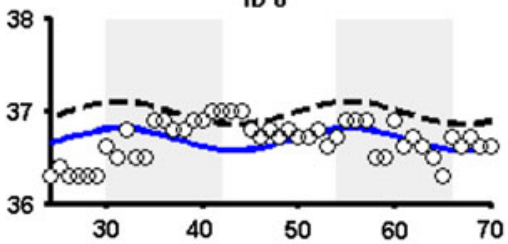

ID 10

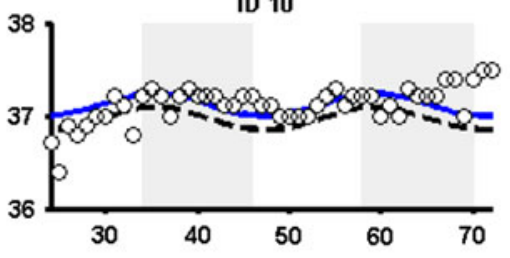

ID 2

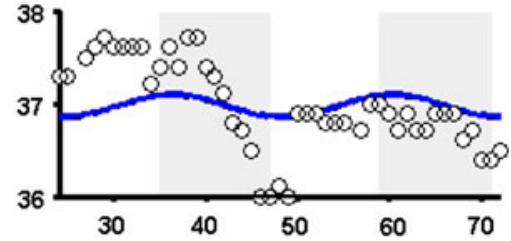

ID 5

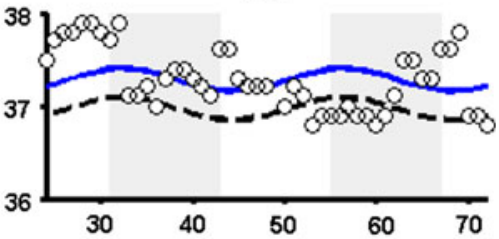

ID 7

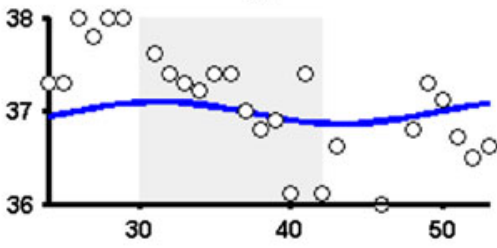

ID 9

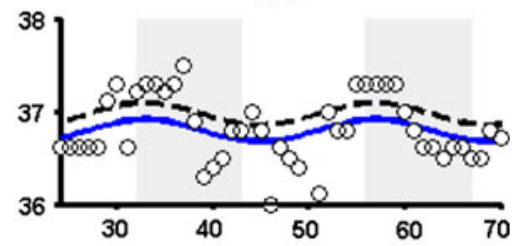

ID 11

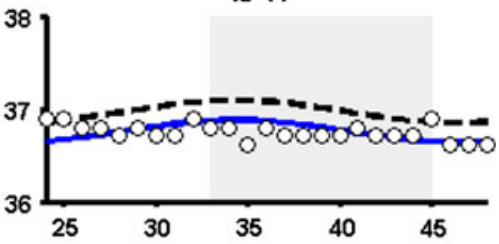

Time, $\mathbf{h}$

Fig. 4 Plot of observed (open circle), population predicted (dashed line) and individual predicted (solid line) body temperatures versus time for the final model (Eq. 7) with circadian rhythms. The shaded area represents the dark period ( 8 p.m. -8 a.m). Time denotes time that has passed since the start of infusion

between the propofol concentrations and BIS score. The $E C_{50}$ equaled $1.98 \mathrm{mg} / \mathrm{l}$, which is in agreement with the $E C_{50}$ of $2.59 \mathrm{mg} / \mathrm{l}$ for the basic model proposed by Peeters et al. [22]. We did not find any statistically significant covariates. In the literature the Sequential Organ Failure Assessments (SOFA) scores was suggested as a covariate that influences the $E C_{50}$ [22]. Unfortunately, we did not collect SOFA scores to confirm these findings. 
Biological rhythmicity is a fundamental regulatory principle in living organisms. All previous animal studies have shown that the maximum hypnotic effect of general anesthetics was generally obtained during the rest phase, which corresponds in humans to the night time. The longest duration of propofol anesthesia in rats occurred during the natural sleep period [16]. Circadian rhythms were observed in rat hepatic enzyme activity with high values in the activity phase and low values in the rest phase [29]. Since propofol is mainly metabolized by hepatic microsomal enzymes, rhythms in propofol concentrations are possible. However, our data suggest lack of daily fluctuations in the steady-state concentrations and metabolic clearance of propofol in intensive care unit patients.

Rhythmicity in propofol sedative effects might also be expected, since natural sleep and anesthesia appear to share a common mechanism [17]. Nelson et al. [17] noted that propofol sedation is not due to generalized inhibition throughout the CNS, but it rather involves actions on specific neuronal pathways, particularly those in the hypothalamus that underlie NREM sleep. Dispersyn et al. observed desynchronization of daily rest-activity rhythm in the days following light propofol anesthesia [30]. Also, the daily variation in GABA-A activity was described in the cerebral cortex of a hamster [31]. Our results did not confirm a circadian fluctuation in propofol sedation. The data obtained in animal studies cannot be extrapolated to ICU patients sedated with prolonged propofol infusion.

Albrecht et al. [32] studied propofol sedative therapy with median frequency monitoring in trauma and non-trauma patients. They noted that sedation in nontrauma patients revealed some circadian patterns. In contrast the authors did not observe any fluctuations in the trauma patients group, where most of them had head injuries. Similarly, Mc Leod et al. [33] observed that the rhythmicity of sedation cannot be seen in some severely ill patients. In our study patients were clinically stable during the study period and those with head injuries were excluded. In the study by Albrecht et al. propofol plasma concentrations to achieve the same level of sedation were highest between 8 a.m. and 4 p.m. and lowest between 11 p.m. and 4 a.m. This is in contradiction to our findings which showed no significant circadian rhythm with respect to BIS score during the constant infusion of propofol.

In our study we noted disturbances in the rhythmicity of the monitored physiological parameters. We recorded blood pressure, heart rate, body temperature and blood oxygen saturation every hour during the second and the third day of the propofol infusion. It is important to examine the circadian blood pressure variations because the natural rhythm alterations are known to influence life expectancy [33-36]. Both heart rate and blood pressure exhibit a strong circadian pattern with the values typically peaking in the morning. Normally, the blood pressure rises rapidly in the early morning hours due to increased plasma renin activity and increased secretion of catecholamines. In the late morning (at about 9-10 a.m.) the blood pressure reaches its peak [34]. After that blood pressure declines and is the lowest between midnight and 3 a.m. In our study no BP rhytmicity was detected; suggesting severe disturbance from the natural pattern. For healthy subjects the heart rate reaches its peak around 11-12 a.m. and a nightly fall by about $10 \%$ is observed [35, 37]. Paul et al. [35] noted a minor nocturnal fall in the ICU patients heart rate, the day-night difference equaled 3.4 beats $/ \mathrm{min}$ ( $3.2 \%$ of the mean heart rate value). This is comparable to our findings. 
A normal circadian pattern in the body temperature has an acrophase at about 5 p.m. with an amplitude of $0.38^{\circ} \mathrm{C}$ around the mean of $36.6^{\circ} \mathrm{C}$. The minimum of body temperature is observed in the early morning, around 4 a.m. [38, 39]. The administration of sedatives is thought to suppress temperature rhythms directly by an effect on oscillators, or indirectly through an effect on consciousness [33]. Paul et al. [35] did not note any normal circadian pattern in body temperatures of the intensive care patients, although the authors observed a small decrease at night. The mean day value of the body temperature was $37.9 \pm 1.3^{\circ} \mathrm{C}$, whereas the mean night value was $37.6 \pm 1.3^{\circ} \mathrm{C}$. In our study, similarly to normal human variations, the patients' body temperature tended to be lower in the morning and then increased during the day. We obtained some significant day-night difference in the body temperature profile; however, a low amplitude of the mean body temperature rhythm was noted $\left(0.12^{\circ} \mathrm{C}\right)$.

The limitation of our study was the lack of biochemical monitoring. The variations in all the monitored physiological parameters may be strongly connected with the circadian profiles of circulating melatonin, cortisol, norepinephrine, aldosterone, and other hormones. Some alterations in the concentration of these substances have been observed due to various disease states and sedative agents given $[35,36,40]$.

\section{Conclusions}

The light-dark cycle did not influence propofol pharmacokinetics and pharmacodynamics in intensive care unit patients. According to other monitored parameters rhythm disturbances were observed when compared to the well known rhythmic $24 \mathrm{~h}$ patterns. We observed circadian rhythms for heart rate and body temperature, however with very small amplitude. The lack of night-day difference was noted for both systolic and diastolic blood pressure and blood oxygen saturation. Our results support the hypothesis that endogenous rhythms in critically ill and sedated patients are severely disturbed and desynchronized.

Acknowledgment One of the authors (Paweł Wiczling) was supported by the START fellowship and by a grant from Iceland, Liechtenstein and Norway through the EEA Financial Mechanism via Homing Program from the Foundation for Polish Science.

Open Access This article is distributed under the terms of the Creative Commons Attribution Noncommercial License which permits any noncommercial use, distribution, and reproduction in any medium, provided the original author(s) and source are credited.

\section{References}

1. Rinaldi S, Consales G, Gaudio AR (2006) Sedation monitoring in ICU. Curr Anaesth Crit Care 17:303-315

2. Liu J, Shingh H, White PF (1997) Electroencephalographic bispectral index correlates with intraoperative recall and depth of propofol-induced sedation. Anesth Analg 84:185-189 
3. Glass PS, Bloom M, Kearse L, Rosow C, Sebel P, Manberg P (1997) Bispectral analysis measures sedation and memory effects of propofol, midazolam, isoflurane and alfentanil in healthy volunteers. Anesthesiology 86:836-847

4. Sigl JC, Chamoun NG (1994) An introduction to bispectral analysis for the electroencephalogram. J Clin Monit 10:392-404

5. Johansen JW, Sebel PS (2000) Development and clinical application of electroencephalographic bispectrum monitoring. Anesthesiology 93:1336-1344

6. Doi M, Gajraj J, Mantzaridis H, Kenny GNC (1997) Relationship between calculated blood concentration of propofol and electrophysiological variables during emergence from anaesthesia: comparison of bispectral index, spectral edge frequency, median frequency and auditory evoked potential index. Br J Anaesth 78:180-184

7. Lee GY (2002) Titration of effect site concentration of propofol for conscious sedation in elderly patients. Kor J Anesth 43:198-202

8. Hye-Jin Park, Yong LM, Chong SK, Seong DK, Hee-Soo K (2007) Changes of bispectral index during recovery from general anesthesia with $2 \%$ propofol and remifentanil in children. Pediatr Anesth 17:35-37

9. Leslie K, Sessler DI, Schroeder M, Walters K (1995) propofol blood concentration and the bispectral index predict suppresssion of learning during propofol/epidural anesthesia in volunteers. Anesth Analg 81:1269-1274

10. Simmons LE, Riker RR, Prato BS, Fraser GL (1999) Assessing sedation during intensive care unit mechanical ventilation with the Bispectral index and the Sedation-Agitation Scale. Crit Care Med 27:1499-1504

11. Riker RR, Fraser GL, Simmons LE, Wilkins ML (2001) Validating the Sedation-Agitation scale with the Bispectral index and Visual Analog scale in Adult ICU patients after cardiac surgery. Int Care Med 27:853-858

12. Frenzel D, Greim CA, Sommer C, Bauerle K, Roewer N (2002) Is the bispectral index appropriate for monitoring the sedation level of mechanically ventilated surgical ICU patients? Int Care Med 28:178-183

13. Riess ML, Graefe UA, Goeters C, Van Aken H, Bone HG (2002) Sedation assessment in critically ill patients with bispectral index. Eur J Anaesth 19:18-22

14. Zapantis A, Leong S (2005) Tolerance and withdrawal issues with sedation. Crit Care Nurs Clin North Am 17:211-223

15. Chassard D, Frederic D, Siquueira M, Allaouchiche B, Bosseli E (2007) Chronobiology and anesthesia. Curr Opin Anaesthesiol 20:186-190

16. Challet E, Gourmelen S, Pevet P, Oberling P, Pain L (2007) Reciprocal relationship between general (propofol) anesthesia and circadian time in rats. Neuropsychopharmacology 32:728-735

17. Nelson LE, Guo TZ, Lu J, Saper CB, Franks NP, Maze M (2002) The sedative component of anesthesia is mediated by $\mathrm{GABA}_{\mathrm{A}}$ receptors in an endogenous sleep pathway. Nat Neurosci 5:979-984

18. Plummer GF (1987) Improved method for the determination of propofol in blood by high-performance liquid chromatography with fluorescence detection. J Chromatogr 421:171-176

19. Schüttler J, Ihmsen H (2000) Population pharmacokinetics of propofol. A multicenter study. Anesthesiology 92:727-738

20. Shafer SL (1993) Advances in propofol pharmacokinetics and pharmacodynamics. J Clin Anesth 5:14-21

21. Prins SA, Peeters MY, Houmes RJ, van Dijk M, Knibbe CA, Danhof M, Tibboel D (2005) Propofol $6 \%$ as sedative in children under 2 years of age following major craniofacial surgery. Br J Anaesth 94:630-635

22. Peeters MY, Bras LJ, DeJongh J, Wesselink RM, Aarts LP, Danhof M, Knibbe CA (2008) Disease severity is a major determinant for the pharmacodynamics of propofol in critically ill patients. Clin Pharmacol Ther 83:443-451

23. Sobara C, Armellin G, Bonato R, Callegher L, Giron G (1998) Postoperative sedation with propofol infusion: hemodynamics and pharmacokinetics. Clin Drug Investig 16:431-439

24. Peeters MY, Aarts LP, Boom FA, Bras LJ, Tibboel D, Danhof M, Knibbe CA (2008) Pilot study on the influence of liver blood flow and cardiac output on the clearance of propofol in critically ill patients. Eur J Clin Pharmacol 64:329-334

25. Bailie GR, Cockshott ID, Douglas EJ, Bowles BJ (1992) Pharmacokinetics of propofol during and after long term continuos infusion for maintenance of sedation in ICU patients. Br J Anaesth 68: 486-491 
26. Albanese J, Martin C, Lacarelle B, Saux P, Durand A, Gouin F (1990) Pharmacokinetics of long-term propofol infusion used for sedation in ICU patients. Anesthesiology 73:214-217

27. Barr J, Egan TD, Sandoval NF, Zomorodi K, Cohane C, Gambus PL, Shafer SL (2001) Propofol dosing regimens for ICU sedation based upon an integrated pharmacokinetic-pharmacodynamic model. Anesthesiology 95:324-333

28. Frenkel C, Schuttler J, Ihmsen H, Heye H, Rommelsheim K (1995) Pharmacokinetics and pharmacodynamics of propofol/alfentanil infusion for sedation in ICU patients. Intensive Care Med 21:981-988

29. Furukawa T, Manabe S, Watanabe T, Sharyo S, Mori Y (1999) Sex difference in the daily rhythm of hepatic $\mathrm{P} 450$ monooxygenase acivities in rats is regulated by growth hormone release. Toxicol Appl Pharmacol 161:219-224

30. Dispersyn G, Touitou Y, Coste O, Jouffroy L, Lleu JC, Challet E, Pain L (2009) Desynchronization of daily rest-activity rhythm in the days following light propofol anesthesia for colonoscopy. Clin Pharmacol Ther 85:51-55

31. Kantarewicz BI, Rosenstein RE, Golombek DA, Yannielli PC, Cardinalli DP (1995) Daily variations in GABA receptor function in Syrian hamster cerebral cortex. Neurosci Lett 200:211-213

32. Albrecht S, Frenkel C, Ihmsen H, Schuttler J (1999) A rational approach to the control of sedation in intensive care units patients based on closed-loop control. E Journal Anaesth 16:678-687

33. McLeod G, Wallis C, Dick J, Cox C, Patterson A, Colvin J (1997) Use of $2 \%$ propofol to produce diurnal sedation in critically ill patients. Intensive Care Med 23:428-434

34. Smith DHG (2001) Pharmacology of cardiovascular chronotherapeutic agents. Am J Hypertens 14:296S-301S

35. Paul T, Lemmer B (2007) Disturbance of circadian rhythms in analgosedated intensive care unit patients with and without craniocerebral injury. Chronobiol Int 24:45-61

36. Halhuber MJ, Cornelissen G, Bartter FC, Delea CS, Kreze A, Mikulecky M, Muller-Bohn T, Siegelova J, Dusek J, Schwartkopf O, Halberg F (2002) Circadian urinary glucocorticoid and blood pressure coordination. Scripta Medica 75:139-144

37. Krauchi K, Wirz Justice A (1994) Circadian rhythm of heat production, heart rate and skin and core temperature under unmasking conditions in men. Am J Physiol Regul Integr Comp Physiol 267: $819-829$

38. Smolander J, Harma M, Lindqvist A, Kolari P, Laitinen LA (1993) Circadian variation in peripheral blood flow in relation to core temperature at rest. Eur J Appl Physiol 67:192-196

39. Baehr EK, Revelle W, Eastman CI (2000) Individual differences in the phase and amplitude of the human circadian temperature rhythm: with an emphasis on morningess-eveningness. J Sleep Res 9:117-127

40. Reber A, Huber PR, Ummenhofer W, Gurtler CM, Zurschmiede C, Drewe J, Schneider M (1998) General anesthesia for surgery can influence circulating melatonin during hours. Acta Anaesthesiol Scand 42:1050-1056 